\title{
New radial velocity studies and curve analysis of spectroscopic binary systems CS22964-161, LV Her, RW Lac and HD 34700
}

\author{
K. Ghaderi*, M. H. Baghadam, T. Rostami \\ Department of Science and Engineering, Islamic Azad University, Marivan Branch, Marivan, Iran \\ *E-mail address: K.Ghaderi.60@gmail.com
}

\begin{abstract}
Using measured radial velocity data of four double-lined spectroscopic binary systems CS22964-161, LV Her, RW Lac and HD 34700, we find corresponding orbital and spectroscopic elements via a Probabilistic Neural Network (PNN). Our numerical results are in good agreement with those obtained by others using more traditional methods.
\end{abstract}

Keywords: Stars; binaries; eclipsing - Stars; binaries; spectroscopic

\section{INTRODUCTION}

Analysis of both light and radial velocity (hereafter $V_{R}$ ) curves of binary systems helps us to determine the masses and radii of individual stars. One historically well-known method to analyze the $V_{R}$ curve is that of Lehmann-Filhés [1]. Some other methods were also introduced by Sterne [2] and Petrie [3]. The different methods of the $V_{R}$ curve analysis have been reviewed in ample detail by Karami \& Teimoorinia [4]. Karami \& Teimoorinia [4] also proposed a new non-linear least squares velocity curve analysis technique for spectroscopic binary stars. They showed the validity of their new method to a wide range of different types of binary See Karami \& Mohebi [5-7] and Karami et al. [8].

Artificial Neural Networks have become a popular tool in almost every field of science. In recent years, ANNs have been widely used in astronomy for applications such as star/galaxy discrimination, morphological classification of galaxies, and spectral classification of stars (see Bazarghan et al. [9] and references therein). Following Bazarghan et al. [9], we employ Probabilistic Neural Networks (PNNs). This network has been investigated in ample details by Bazarghan et al. [9]. Probabilistic Neural Network (PNN) is a new tool to derive the orbital parameters of the spectroscopic binary stars.

In the present paper we use a Probabilistic Neural Network (PNN) to find the optimum match to the four parameters of the $V_{R}$ curves of the four double-lined spectroscopic binary systems CS22964-161, LV Her, RW Lac and HD 34700. Our aim is to show the validity of our new method to a wide range of different types of binary.

CS 22964-161 is a double-lined spectroscopic binary that both components are near the metal-poor main-sequence turn off and the spectral types of the stars are not too dissimilar and the orbital period is $\mathrm{P}=252.481$ days [10]. LV Her is a double-lined eclipsing binary and consists of primary and secondary components which has the highest eccentricity. The 
spectral type of systems is F9 V and the orbital period is $\mathrm{P}=18.4359535$ days [11]. RW Lac is a detached, eccentric, EA-type, double-lined eclipsing binary and consists of the hotter, larger, more massive, and more luminous photometric primary and the cooler, smaller, less massive, and less luminous photometric secondary components. The spectral type is G5 and G7 for the primary and the secondary stars and the orbital period is $\mathrm{P}=10.3692046$ days [12]. The young star HD 34700 is a double-lined spectroscopic binary and the components are of very nearly equal mass, temperature, luminosity and similar spectral type: G0 and the orbital period is $\mathrm{P}=23.4877$ days [13].

This paper is organized as follows. In Sect. 2, we introduce a Probabilistic Neural Network (PNN) to estimate the four parameters of the $V_{R}$ curve. In Sect. 3, the numerical results are reported, while the conclusions are given in Sect. 4.

\section{2. $V_{R}$ CURVE PARAMETERS ESTIMATION BY THE PROBABILISTIC NEURAL NETWORK (PNN)}

Following Smart [14], the $V_{R}$ of a star in a binary system is defined as follows

$$
\mathrm{V}_{\mathrm{R}}=\gamma+\mathrm{K}[\cos (\theta+\omega)+\mathrm{ecos} \omega]
$$

where $\gamma$ is the $V_{R}$ of the center of mass of system with respect to the sun. Also $K$ is the amplitude of the $V_{R}$ of the star with respect to the center of mass of the binary. Furthermore $\theta, \omega$ and $\mathrm{e}$ are the angular polar coordinate (true anomaly), the longitude of periastron and the eccentricity, respectively.

Here we apply the PNN method to estimate the four orbital parameters, $\gamma, \mathrm{K}$, e and $\omega$ of the $V_{R}$ curve in Eq. (1). In this work, for the identification of the observational $V_{R}$ curves, the input vector is the fitted $V_{R}$ curve of a star. The PNN is first trained to classify $V_{R}$ curves corresponding to all the possible combinations of $\gamma, \mathrm{K}$, e and $\omega$. For this one can synthetically generate $V_{R}$ curves given by Eq. (1) for each combination of the parameters:

$-100 \leq \gamma \leq 100$ in steps of 1 ;

- $1 \leq \mathrm{K} \leq 300 \quad$ in steps of 1 ;

- $0 \leq \mathrm{e} \leq 1 \quad$ in steps of 0.001 ;

- $0 \leq \omega \leq 360^{\circ} \quad$ in steps of 5 .

This gives a very big set of $\mathrm{k}$ pattern groups, where $\mathrm{k}$ denotes the number of different $V_{R}$ classes, one class for each combination of $\gamma, K$, e and $\omega$. Since this very big number of different $V_{R}$ classes leads to some computational limitations, hence one can first start with the big step sizes. Note that from Petrie [3], one can guess $\gamma, \mathrm{K}$, e from a $\mathrm{V}_{\mathrm{R}}$ curve. This enable one to limit the range of parameters around their initial guesses. When the preliminary orbit was derived after several stages, then one can use the above small step sizes to obtain the final orbit. The PNN has four layers including input, pattern, summation, and output layers, respectively (see Fig. 5 in Bazarghan et al. [9]). When an input vector is presented, the pattern layer computes distances from the input vector to the training input vectors and produces a vector whose elements indicate how close the input is to a training input. 
The summation layer sums these contributions for each class of inputs to produce as its net output a vector of probabilities. Finally, a competitive transfer function on the output layer picks the maximum of these probabilities, and produces a 1 for that class and a 0 for the other classes $[15,16]$. Thus, the PNN classifies the input vector into a specific $\mathrm{k}$ class labeled by the four parameters $\gamma, \mathrm{K}$, e and $\omega$ because that class has the maximum probability of being correct.

\section{NUMERICAL RESULTS}

Here, we use the PNN to derive the orbital elements for the four different double-lined spectroscopic systems CS22964-161, LV Her, RW Lac and HD 34700. Using measured $V_{R}$ data of the two components of these systems obtained by Thompson et al. [10] for CS22964161, Torres et al. [11] for LV Her, Sandberg Lacy et al. [12] for RW Lac, Torres [13] for HD 34700, the fitted velocity curves are plotted in terms of the phase in Figs. 1 to 4.

The orbital parameters obtaining from the PNN for CS22964-161, LV Her, RW Lac and HD 34700 are tabulated in Tables 1, 3, 5 and 7, respectively. Tables show that the results are in good accordance with the those obtained by Thompson et al. [10] for CS22964-161, Torres et al. [11] for LV Her, Sandberg Lacy et al. [12] for RW Lac, Torres [13] for HD 34700.

Note that the Gaussian errors of the orbital parameters in Tables 1, 3, 5 and 7 are the same selected steps for generating $\mathrm{V}_{\mathrm{R}}$ curves, i.e. $\Delta \gamma=1, \Delta \mathrm{K}=1, \Delta \mathrm{e}=0.001$ and $\Delta \omega=5$. These are close to the observational errors reported in the literature. Regarding the estimated errors, following Specht [16], the error of the decision boundaries depends on the accuracy with which the underlying Probability Density Functions (PDFs) are estimated. Parzen [17] proved that the expected error gets smaller as the estimate is based on a large data set.

This definition of consistency is particularly important since it means that the true distribution will be approached in a smooth manner. Specht [16] showed that a very large value of the smoothing parameter would cause the estimated errors to be Gaussian regardless of the true underlying distribution and the misclassification rate is stable and does not change dramatically with small changes in the smoothing parameter.

The combined spectroscopic elements including $m_{p} \sin ^{3} i, m_{s} \sin ^{3} i,\left(m_{p}+m_{s}\right) \sin ^{3} i$, $\left(a_{p}+a_{s}\right) \sin i$ and $\frac{m_{s}}{m_{p}}$ are calculated by substituting the estimated parameters $K$, e and $\omega$ in to Eqs. (3), (15) and (16) in Karami and Teimoorinia [4].

The results obtained for the four systems are tabulated in Tables 2, 4, 6 and 8 show that our results are in good agreement with the those obtained by Thompson et al. [10] for CS22964-161, Torres et al. [11] for LV Her, Sandberg Lacy et al. [12] for RW Lac, Torres [13] for HD 34700, respectively.

Here the errors of the combined spectroscopic elements in Tables 2, 4, 6 and 8 are obtained by the help of orbital parameters errors. See again Eqs. (3), (15) and (16) in Karami and Teimoorinia [4]. 


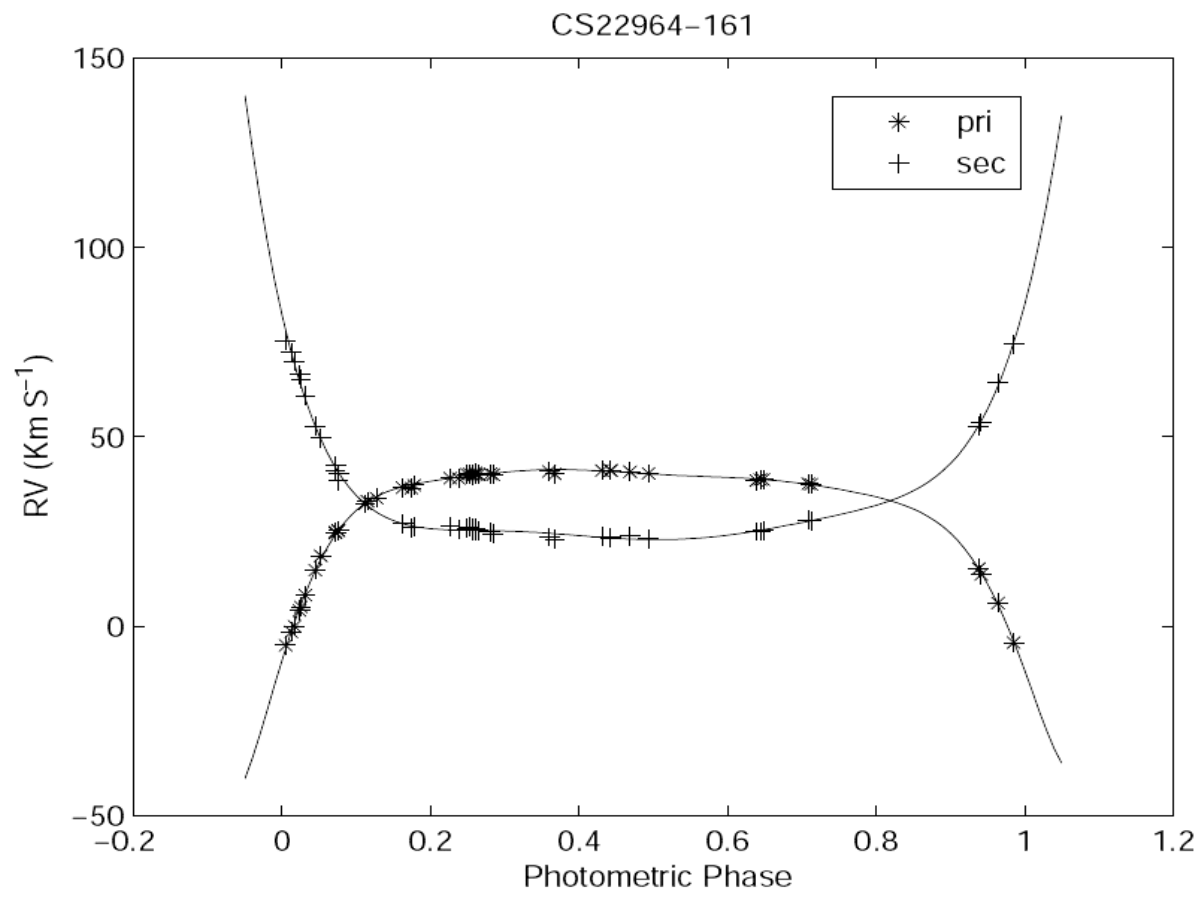

Fig. 1. Radial velocities of the primary and secondary components of CS22964-161plotted against the phase. The observational data have been measured by Thompson et al. [10].

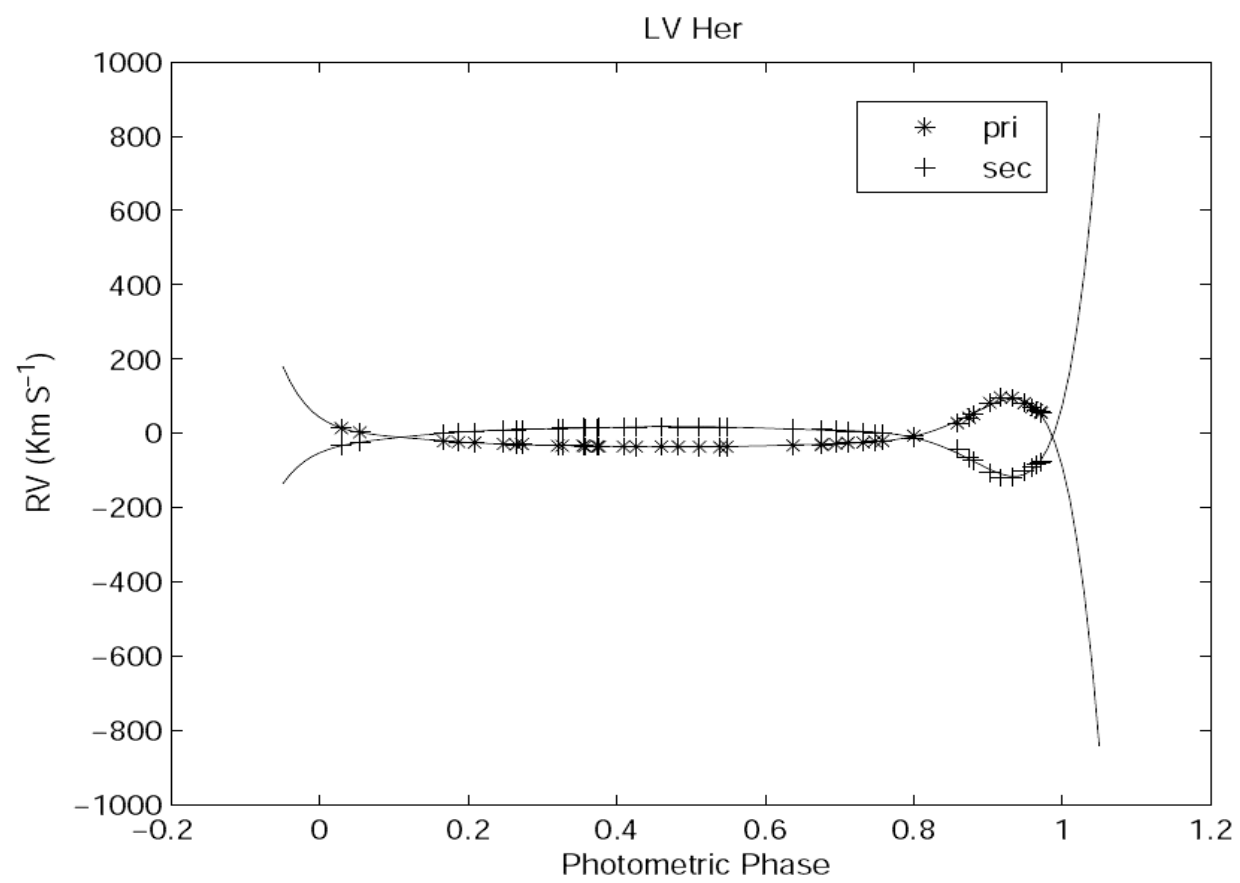

Fig. 2. Radial velocities of the primary and secondary components of LV Her plotted against the phase. The observational data have been measured by Torres et al. [11]. 


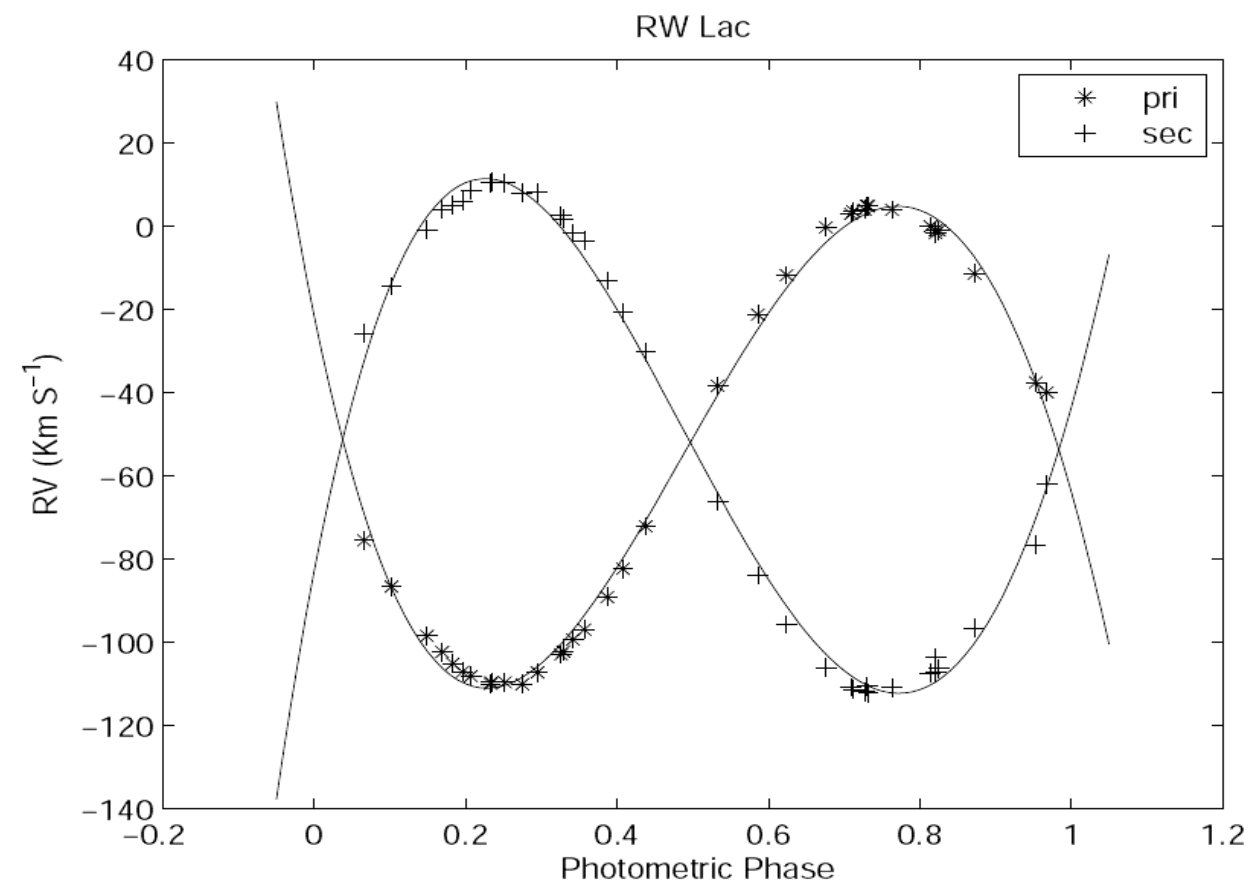

Fig. 3. Radial velocities of the primary and secondary components of RW Lac plotted against the phase. The observational data have been measured by Sandberg Lacy et al. [12].

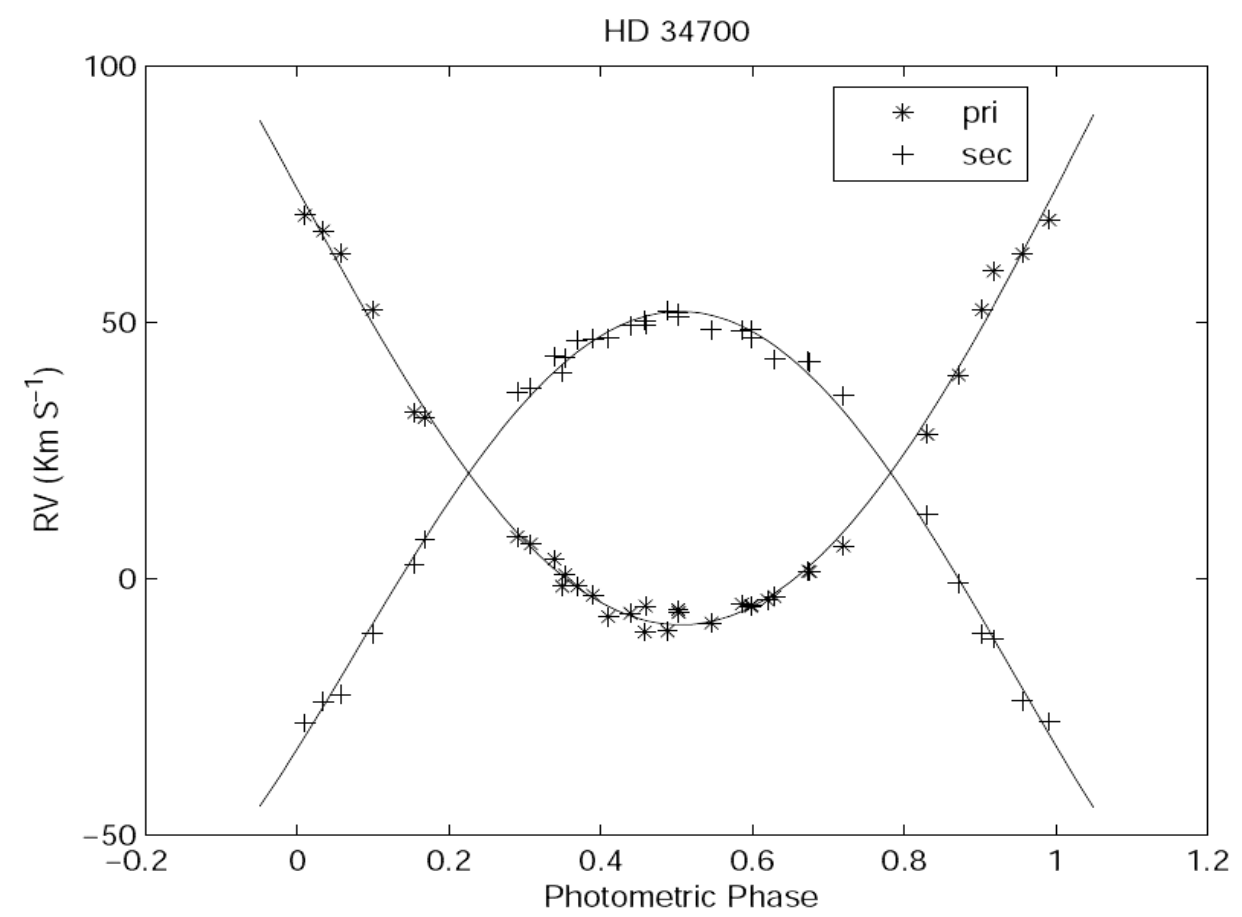

Fig. 4. Radial velocities of the primary and secondary components of HD 34700 plotted against the phase. The observational data have been measured by Torres [13]. 
Table 1. Orbital parameters of CS22964-161.

\begin{tabular}{lll}
\hline \hline & This Paper & Thompson et al. [9] \\
\hline$\gamma\left(k m s^{-1}\right)$ & $33 \pm 1$ & $32.85 \pm 0.05$ \\
$K_{p}\left(k m s^{-1}\right)$ & $24 \pm 1$ & $23.65 \pm 0.10$ \\
$K_{s}\left(k m s^{-1}\right)$ & $27 \pm 1$ & $26.92 \pm 0.13$ \\
$e$ & $0.655 \pm 0.001$ & $0.6564 \pm 0.0024$ \\
$\omega\left(^{\circ}\right)$ & $185 \pm 5$ & $188.54 \pm 0.39$ \\
& & \\
\hline \hline
\end{tabular}

Table 2. Combined spectroscopic elements of CS22964-161.

\begin{tabular}{lll}
\hline \hline Parameter & This Paper & Thompson et al. [9] \\
\hline$m_{p} \sin ^{3} i / M_{\odot}$ & $0.7926 \pm 0.0001$ & $0.773 \pm 0.009$ \\
$m_{s} \sin ^{3} i / M_{\odot}$ & $0.7045 \pm 0.0001$ & $0.680 \pm 0.007$ \\
$\left(m_{p}+m_{s}\right) \sin ^{3} i / M_{\odot}$ & $1.4971 \pm 0.0002$ & - \\
$a_{p} \sin i / R_{\odot}$ & $90.4248 \pm 3.6640$ & - \\
$a_{s} \sin i / R_{\odot}$ & $101.7279 \pm 3.6510$ & - \\
$\left(a_{p}+a_{s}\right) \sin i / R_{\odot}$ & $192.1527 \pm 7.3150$ & $190.32 \pm 0.65$ \\
$m_{s} / m_{p}$ & $0.8889 \pm 0.0052$ & - \\
\hline \hline
\end{tabular}

Table 3. Orbital parameters of LV Her.

\begin{tabular}{lll}
\hline \hline & This Paper & Torres et al. [10] \\
\hline$\gamma\left(k m s^{-1}\right)$ & $-10 \pm 1$ & $-10.278 \pm 0.094$ \\
$K_{p}\left(k m s^{-1}\right)$ & $67 \pm 1$ & $67.24 \pm 0.19$ \\
$K_{s}\left(k m s^{-1}\right)$ & $68 \pm 1$ & $68.59 \pm 0.27$ \\
$e$ & $0.613 \pm 0.001$ & $0.61273 \pm 0.00073$ \\
$\omega\left(^{\circ}\right)$ & $350 \pm 5$ & $352.20 \pm 0.24$ \\
& & \\
\hline \hline
\end{tabular}


Table 4. Combined spectroscopic elements of LV Her.

\begin{tabular}{lll}
\hline \hline Parameter & This Paper & Torres et al. [10] \\
\hline$m_{p} \sin ^{3} i / M_{\odot}$ & $1.1675 \pm 0.0003$ & $1.193 \pm 0.010$ \\
$m_{s} \sin ^{3} i / M_{\odot}$ & $1.1503 \pm 0.0003$ & $1.1697 \pm 0.0080$ \\
$\left(m_{p}+m_{s}\right) \sin ^{3} i / M_{\odot}$ & $2.3177 \pm 0.0006$ & - \\
$a_{p} \sin i / 10^{6} k m$ & $13.4267 \pm 0.1872$ & $13.471 \pm 0.037$ \\
$a_{s} \sin i / 10^{6} k m$ & $13.6271 \pm 0.1870$ & $13.743 \pm 0.054$ \\
$\left(a_{p}+a_{s}\right) \sin i / R_{\odot}$ & $38.8339 \pm 0.5372$ & $39.119 \pm 0.095$ \\
$m_{s} / m_{p}$ & $0.9853 \pm 0.0002$ & $0.9803 \pm 0.0047$ \\
\hline \hline
\end{tabular}

Table 5. Orbital parameters of RW Lac.

\begin{tabular}{lll}
\hline \hline & This Paper & Sandberg Lacy et al. [11] \\
\hline$\gamma\left(k m s^{-1}\right)$ & $-52 \pm 1$ & $-51.79 \pm 0.08$ \\
$K_{p}\left(k m s^{-1}\right)$ & $58 \pm 1$ & $57.44 \pm 0.10$ \\
$K_{s}\left(k m s^{-1}\right)$ & $61 \pm 1$ & $61.27 \pm 0.19$ \\
$e$ & $0.009 \pm 0.001$ & $0.0098 \pm 0.0010$ \\
$\omega\left(^{\circ}\right)$ & $180 \pm 5$ & $183 \pm 11$ \\
\hline \hline
\end{tabular}

Table 6. Combined spectroscopic elements of RW Lac.

\begin{tabular}{lll}
\hline \hline Parameter & This Paper & Sandberg Lacy et al. [11] \\
\hline$m_{p} \sin ^{3} i / M_{\odot}$ & $0.9279 \pm 0.005$ & $0.928 \pm 0.006$ \\
$m_{s} \sin ^{3} i / M_{\odot}$ & $0.8823 \pm 0.0005$ & $0.869 \pm 0.004$ \\
$\left(m_{p}+m_{s}\right) \sin ^{3} i / M_{\odot}$ & $1.8101 \pm 0.0010$ & - \\
$a_{p} \sin i / R_{\odot}$ & $11.8767 \pm 0.2047$ & - \\
$a_{s} \sin i / R_{\odot}$ & $12.4910 \pm 0.2047$ & - \\
$\left(a_{p}+a_{s}\right) \sin i / R_{\odot}$ & $24.3677 \pm 0.4093$ & - \\
$m_{s} / m_{p}$ & $0.9508 \pm 0.0009$ & $0.9375 \pm 0.0035$ \\
\hline \hline
\end{tabular}


Table 7. Orbital parameters of HD 34700.

\begin{tabular}{lll}
\hline \hline & This Paper & Torres [12] \\
\hline$\gamma\left(k m s^{-1}\right)$ & $21 \pm 1$ & $21.03 \pm 0.18$ \\
$K_{p}\left(k m s^{-1}\right)$ & $39 \pm 1$ & $38.83 \pm 0.42$ \\
$K_{s}\left(k m s^{-1}\right)$ & $40 \pm 1$ & $39.33 \pm 0.34$ \\
$e$ & $0.251 \pm 0.001$ & $0.2501 \pm 0.0068$ \\
$\omega\left(^{\circ}\right)$ & $355 \pm 5$ & $358.1 \pm 1.6$ \\
& & \\
\hline \hline
\end{tabular}

Table 8. Combined spectroscopic elements of HD 34700.

\begin{tabular}{lll}
\hline \hline Parameter & This Paper & Torres [12] \\
\hline$m_{p} \sin ^{3} i / M_{\odot}$ & $0.5510 \pm 0.0003$ & $0.531 \pm 0.011$ \\
$m_{s} \sin ^{3} i / M_{\odot}$ & $0.5372 \pm 0.0003$ & $0.524 \pm 0.012$ \\
$\left(m_{p}+m_{s}\right) \sin ^{3} i / M_{\odot}$ & $1.0882 \pm 0.0006$ & - \\
$a_{p} \sin i / 10^{6} k m$ & $12.1992 \pm 0.3095$ & $12.14 \pm 0.13$ \\
$a_{s} \sin i / 10^{6} k m$ & $12.5120 \pm 0.3094$ & $12.30 \pm 0.11$ \\
$\left(a_{p}+a_{s}\right) \sin i / R_{\odot}$ & $35.4712 \pm 0.8885$ & $35.12 \pm 0.25$ \\
$m_{s} / m_{p}$ & $0.9750 \pm 0.007$ & $0.987 \pm 0.014$ \\
\hline \hline
\end{tabular}

\section{CONCLUSIONS}

A Probabilistic Neural Network to derive the orbital elements of spectroscopic binary stars was applied. PNNs are used in both regression (including parameter estimation) and classification problems. However, one can discretize a continuous regression problem to such a degree that it can be represented as a classification problem $[15,16]$, as we did in this work.

Using the measured $V_{R}$ data of CS22964-161, LV Her, RW Lac and HD 34700 obtained by Thompson et al. [10], Torres et al. [11], Sandberg Lacy et al. [12] and Torres [13], respectively, we find the orbital elements of these systems by the PNN. Our numerical results shows that the results obtained for the orbital and spectroscopic parameters are in good agreement with those obtained by others using more traditional methods.

This method is applicable to orbits of all eccentricities and inclination angles. In this method the time consumed is considerably less than the method of Lehmann-Filhés. It is also 
more accurate as the orbital elements are deduced from all points of the velocity curve instead of four in the method of Lehmann-Filhés. The present method enables one to vary all of the unknown parameters $\gamma, \mathrm{K}$, e and $\omega$ simultaneously instead of one or two of them at a time. It is possible to make adjustments in the elements before the final result is obtained. There are some cases, for which the geometrical methods are inapplicable, and in these cases the present one may be found useful. One such case would occur when observations are incomplete because certain phases could have not been observed. Another case in which this method is useful is that of a star attended by two dark companions with commensurable periods. In this case the resultant velocity curve may have several unequal maxima and the geometrical methods fail altogether.

\section{References}

[1] R. Lehmann-Filhés, $A N 136$ (1984) 17.

[2] T. E. Sterne, PNAS 27 (1941) 175.

[3] R. M. Petrie, "Astronomical Techniques," Ed., W. A. Hiltner, University of Chicago Press, Chicago (1960).

[4] K. Karami, H. Teimoorinia, Ap \& SS 311 (2007) 435.

[5] K. Karami, R. Mohebi, ChJAA 7 (2007) 668.

[6] K. Karami, R. Mohebi, JApA 28 (2007) 217.

[7] K. Karami, R. Mohebi, JApA 30 (2009) 153.

[8] K. Karami, R. Mohebi, M. M. Soltanzadeh, Ap \& SS 318 (2008) 69.

[9] M. Bazarghan, H. Safari, D. E. Innes, E. Karami, S. K. Solanki, $A \&$ \& 492 (2008) L13.

[10] I. B. Thompson, I. I. Ivans, S. Bisterzo, C. Sneden, R. Gallino, S. Vauclair, G. S. Burley, S. A. Shectman, G. W. Preston, AJ 677 (2008) 556.

[11] G. Torres, C. H. Sandberg Lacy, A. Claret, AJ 138 (2009) 1622.

[12] C. H. Sandberg Lacy, G. Torres, A. Claret, L. P. Ribeiro Vaz, AJ 130 (2005) 2838.

[13] G. Torres, $A J 127$ (2007) 1187.

[14] W. M. Smart, "Textbook on Spherical Astronomy," Sixth Ed., Revised by R. M. Green, 1990, Cambridge Univ. Press, 360.

[15] D. F. Specht, "in Proc IEEE International Conference on Neural Networks" 1988, 525.

[16] D. F. Specht, Neural Networks 3 (1990) 109.

[17] E. Parzen, Annals of Mathematical Statistics 33 (1962) 1065. 